\title{
Simultaneous Operation of Multiple Collocated Radios and the Scanning Problem
}

\author{
Michel Barbeau \\ Carleton University, School of Computer Science \\ 5302 Herzberg Building, 1125 Colonel By Drive, Ottawa, Ontario, K1S 5B6, Canada \\ E-mail: barbeau@scs.carleton.ca
}

\begin{abstract}
Simultaneous operation of multiple collocated radios refers to the capability of a wireless device to operate at the same time into several modes, standards and frequencies. For instance, most of the mobile phones are able and try to operate in multiple modes. Each wireless communication unit is called a radio interface. The software defined radio technology facilitates the integration and simultaneous operation of several radio interfaces in the same hardware device. Simultaneous operation produces interference and hardware conflicts, which can be addressed using a turn-based model. Each radio interface operates periodically for an interval of time. In this paper, the focus is on one radio interface that operates periodically for a short interval of time. The radio interface scans channels to uncover beacons periodically sent by transmitters. The goal of the scanning activity is to uncover the beacons within the shortest possible time. We call this the scanning problem. We model and compare four strategies to solve the scanning problem: sequential scanning, sliding-window scanning, pseudo-concurrent scanning and truly-concurrent scanning.
\end{abstract}

\section{INTRODUCTION}

Simultaneous operation of multiple collocated radios refers to the capability of a wireless device to operate at the same time into several modes, standards and frequencies, e.g., simultaneous operation in the $\mathrm{WiFi} / 802.11$, WiMAX/802.16 and Bluetooth/802.15.1 bands or simultaneous operation of several $\mathrm{WiFi} / 802.11$ wireless cards on the same computer. Each wireless communication unit is called a radio interface. The software defined radio technology facilitates the integration and simultaneous operation of several radio interfaces in the same hardware device. Collocated radio interfaces share resources partially (e.g., an antenna) or entirely (e.g., a whole software defined radio). During simultaneous operation, radio interfaces generate hardware conflicts and interference that degrades the performance. Adequate interleaving of the operations can address these problems. Each radio interface is scheduled to operate in turn for an interval of time. In this paper, we assume a turn-based model. Each radio interface operates periodically for a interval of time.

The focus is on one radio interface that scans channels to receive beacons sent periodically by transmitters. In the single transmitter version, the receiver scans the transmitter's channels for periodic short intervals of time. The variable $C$ denotes the length of the receive period. The variable $R$ denotes the time during which the receiver is listening and can receive the signal from the transmitter, $R \leq C$. The transmitter

978-1-4577-2028-4/11/ \$26.00 (c) 2011 IEEE sends periodically, using broadcast, a beacon signal that aims to announce its existence and willingness to be discovered. The period of the broadcast is $B$. The duration of a beacon is $T$, with $T \leq R$. The discovery activity succeeds when the beacon transmission $T$ falls within the receive window $R$. The receiver starts periodic scanning at time $t_{0}$. The first beacon transmission occurs at time $t, t_{0} \leq t \leq B-T$. The goal of the scanning activity is to succeed within the shortest possible time. We call this the scanning problem. For the sake of simplicity, in the sequel $R(T)$ denotes both the receive (transmit) window and its length in time.

We develop probabilistic models for four strategies that solve the scanning problem: sequential scanning, slidingwindow scanning, pseudo-concurrent scanning and trulyconcurrent scanning. Using the probabilistic models and real world parameters, the four strategies are compared.

The problematic of simultaneous multi-radio operation is reviewed in Section II. Work related to the scanning problem is reviewed in Section III. Our contributions, i.e., the probabilistic models and comparisons, are presented in Section IV. We conclude with Section V.

\section{Simultaneous Multi-Radio Operation}

The type of work addressed in this paper is different from the work about the coexistence of heterogeneous wireless networks where radios do interfere but aren't necessarily collocated, such as the work of Jing and Raychaudhuri on WiFi and WiMAX [1]. Zhu and Yin published a tutorial paper on issues related to the operation of multiple collocated radios, in the context of 802.16 networks [2]. Interference and hardware conflict are the two main issues. A transmitter of a radio interface may interfere because of imperfect filtering or noise produced by its local oscillator falling into the bands of receivers of other collocated radio interfaces. In contrast to interfering non collocated radios, interfering signals of collocated radios travel by electrical conduction rather than electromagnetic propagation. The issue of interference in a multiple radio interface device is explored in more depth in a paper by Zhu et al. [3]. Work is geared towards the elimination of interference by type, i.e., transmitter noise, receiver blocking and inter modulation. A receiver is trapped into a blocking state when a strong signal in an adjacent channel degrades reception of the signal in the channel of interest. Konrad et al. have modeled the receiver blocking con- 
dition and quantified resulting signal degradation [4]. Huang et al. addressed the transmitter noise and receiver blocking issues with hardware design [5]. With the goal of minimizing interference, simultaneous WiFi/802.11b and WiMAX/802.16a operation over the same frequencies has been studied by Zhu and Markovits [6]. Coordination information is exchanged over a common channel.

Multiple integrated radios may share components (e.g., processor, power supply, antenna) and concurrent operation creates hardware conflicts. To cope with potential hardware conflicts, there are two concurrency models: true and perceived. With true concurrency, the collocated radio interfaces are independent. They are designed such that no hardware sharing is required. All can operate at the same time. Interference isolation is, however, a challenge. With perceived concurrency, the collocated radio interfaces operate according to a time sharing and scheduling model. Each radio interface is constrained to operate during allocated time intervals. Three perceived concurrency design principles have been defined: predictability, compressibility and flexible scheduling. Predictability means that each radio interface knows in advance when the other collocated radio interfaces are operating. Design for predictability is important for interference and hardware conflict avoidance. Compressibility aims at minimizing the time during which each radio interface is active and doing the maximum during the allocated time intervals. Compressibility can be achieved with time division-based transmission techniques that reduce the actual length of each transmission to short intervals. Flexibility refers to the ability to reschedule radio activities, which could be for using better available time intervals. Compressibility and flexibility assist with the scheduling of more radio activities in a given time interval.

In this paper, the focus is on the time sharing approach for multiple radio interface devices. Radio operations are scheduled in the time domain. Each radio interface gets exclusive time intervals for operation. The standard IEEE 802.15.2 describes time sharing collaborative mechanisms for the coexistence of heterogeneous radio interfaces in the same device [7]. The emphasis is on the collaboration of radio interfaces operating over the same unlicensed frequencies, i.e., the IEEE 802.15 .1 and $802.11 \mathrm{~b}$ systems (there are also standardization efforts for other wireless system combinations). Operation of a radio interface may alternate or be granted upon request according to the needs. In this paper, we assume the alternate model. Arousing questions are what activities can be done during the intervals granted to a radio interface? How to optimize the use of the intervals? For instance, Zhu et al. did consider a Bluetooth and $\mathrm{WiFi} / 802.11$ multi radio device and determined the number of frames that can be sent during the WiFi intervals [3]. Adya et al. have developed a protocol that coordinates the operation of multiple collocated WiFi/802.11 wireless network cards [8]. The goal is to improve the TCP throughput. When multiple wireless cards are available on a node, the protocol decides which card to use based on a channel quality metric. On a periodic basis,

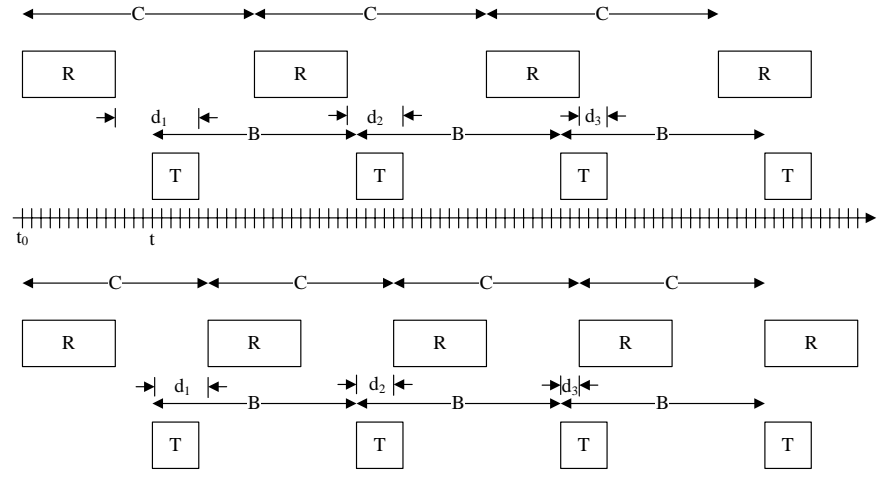

Fig. 1. Sequential scanning (upper part $C>B$, lower part $C<B$ ).

the quality of each channel is evaluated by sending probes and measuring the latency. Xue and Yang modeled a tworadio interface device [9]. Simultaneous receive is possible, but transmission is enabled for only one radio interface at time, with preemptive capability for one of them. Transmission on the non preemptive radio interface is possible only when the preemptive one doesn't need to transmit. The activities of the preemptive radio interface are modeled with a Markov birthdeath process. The performance of scheduling and networking coding strategies are analyzed.

\section{The Scanning Problem}

We hereafter concentrate on radio interfaces that use their time intervals for scanning. In a patent application, Zhu and Markovits proposed a solution to the scanning problem [6]. The model of Zhu and Markovits is pictured in Figure 1 [6]. The periodic intervals granted to a radio interface are used in the receive mode. There are two strategies: sequential scanning and pseudo-concurrent scanning. With sequential scanning, the receiver repeatedly scans the channel of the transmitter until the beacon transmission $T$ falls within the receive window $R$. The scanning procedure starts at time $t_{0}$. From $t_{0}$, the first beacon occurs at time $t$. The receive window begins at the start of each new cycle. For instance, if $t_{0} \leq t \leq R-T$, then one scanning cycle is required. If $t>R-T$, then more than one cycle is required. If we assume that $C>B$, then the time delay between the right edge of $R$ (the end of $R$ ) and right edge of $T$ (the end of $T$ ), represents for how long $T$ is lagging $R$. The lag is denoted as $d_{1}$, in the upper part of Figure 1. We observe that this delay is shorter from one cycle to another until $T$ actually falls within $R$. The relative progress from one cycle to another is the difference $C-B$. In the example of Figure 1, the receiver discovers the transmitter in the fourth scanning interval (upper part).

If we assume that $C<B$, then the time delay between the left edge of $R$ (the start of $R$ ) and left edge of $T$ (the start of $T$ ), represents for how long $T$ is leading $R$. This is denoted as delay $d_{1}$, in the lower part of Figure 1. This delay is shorter from one cycle to another until $T$ falls within the receive window $R$. The relative progress from one cycle to another is the difference $B-C$. Zhu and Markovits do not 


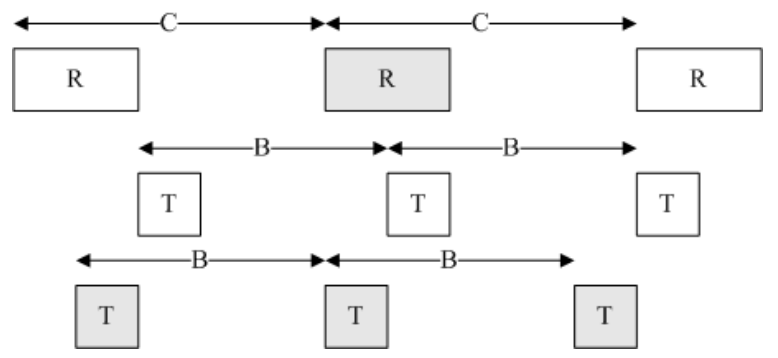

Fig. 2. Pseudo-concurrent scanning $(C>B$ or $C<B)$.

cover the case where $C=B$. We do analyze the case in Subsection IV-B.

The pseudo-concurrent scanning strategy is illustrated in Figure 2. There are several channels. The receiver scans channels until it hears the beacon of a transmitter in each channel. Channels are scanned in groups of $m$. The receiver listens from the first to the $m$-th channel, one after the other from cycle to cycle. In Figure 2, there are two channels: white and gray. The group size $m$ is equal to two. The receiver alternates between the white and gray channels. It discovers a beacon in the gray channel in the second cycle and a beacon in the white channel in the third cycle.

Assuming $C \neq B$, Zhu and Markovits suggest defining the group size as:

$$
m=\left\lfloor\frac{R}{|C-B|}\right\rfloor
$$

The rational behind this logic is that each time a given individual channel is scanned, a different portion of the interval $B$ is received. That is, the receive window $R$ is shifted, with respect to the interval $B$, from on cycle to the other. Indeed, each new cycle shifts the interval $B$ by a relative time delay $|C-B|$. For $R$ to cover a new portion of the interval $B$, we listen to a channel every $m$ cycles, with $m$ defined as in Equation 1. Eventually, the whole interval $B$ is covered. Hence, the minimum number of times $p$ each individual channel must be scanned to cover the full interval $B$ (assuming $C \neq B$ ):

$$
p=\left\lceil\frac{B-R}{m \times|C-B|}+1\right\rceil
$$

which is equivalent to $\lceil B / R\rceil$. The actual number of channels may be greater than $m$, for example $n$. The number of required rounds is:

$$
p \times m \times\left\lceil\frac{n}{m}\right\rceil
$$

With $m$ equals to one, a sequential scanning is done, channel by channel, and the number of needed cycles is (assuming $C \neq B$ ):

$$
n \times\left\lceil\frac{B-R}{|C-B|}+1\right\rceil
$$

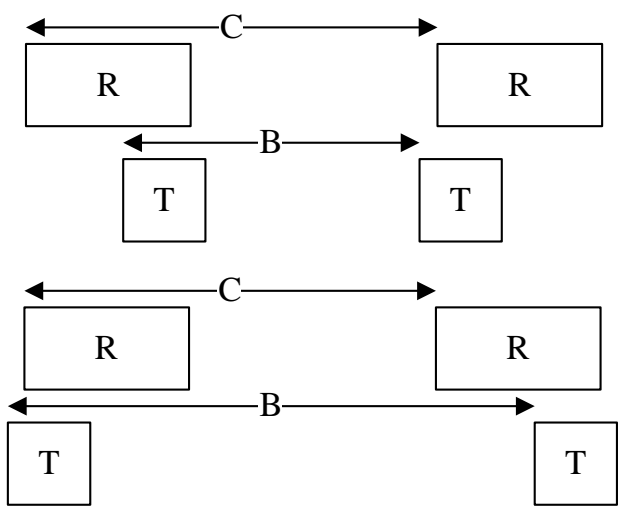

Fig. 3. Cases of rollover, where the assumption $R-|C-B| \geq 2 T$ does not hold.

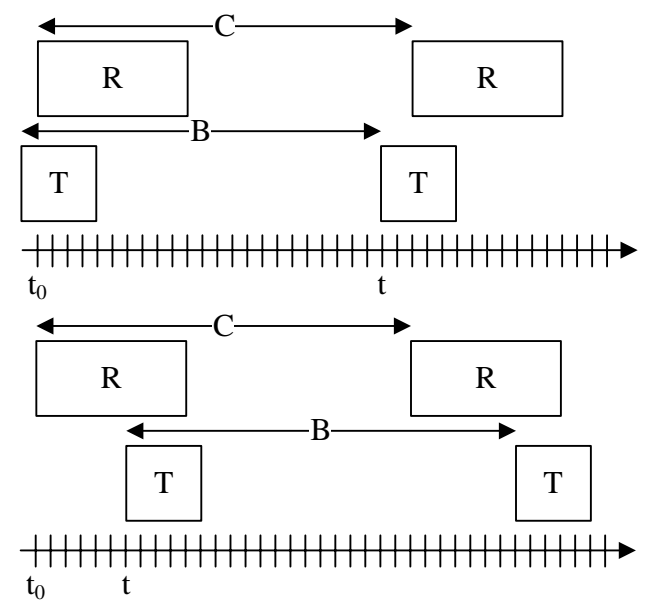

Fig. 4. Worst cases of sequential scanning (upper part $C>B$, lower part $C<B$ ).

\section{Probabilistic Models of Scanning Strategies}

We analyze four strategies to solve the scanning problem. We construct probabilistic models for the sequential scanning and pseudo-concurrent scanning strategies, according to their definition in Ref. [6]. We define and model the sliding-window scanning and truly-concurrent scanning strategies.

\section{A. Sequential Scanning}

We develop a discrete time probabilistic model of sequential scanning. We assume that $R-|C-B| \geq 2 T$. If $C>B$, then this assumption means that if $T$ is lagging $R$, i.e., $t+T>R$, then it not possible for $T$ to rollover and to lead at the next cycle (as pictured in the first part of the Figure 3). Similarly, if $C<B$, then this means that if $T$ is leading $\mathrm{R}$, it is not possible for $T$ to rollover and to lag at the next cycle (as pictured in the second part of the Figure 3).

The worst cases, i.e., when the scanning time until success is the longest, occurs under the following conditions, see Figure 4. If $C>B$, then the worst case is when $t=B-\epsilon$, with $\epsilon>0$ a short delay. If $C<B$, then the worst case is when $t=R-T+\epsilon$. 
Let the symbol $k_{t}$ denote the number of cycles required until a beacon transmission falls within the receive window $R$. This is dependent on $t$, which ranges is $0 \ldots B$. There are two cases. If $C>B$, then the number of cycles corresponds to the ceiling of the following ratio plus one. The ratio is the time difference between the right edge of $T$ (is at time $t+T$ ) and right edge of $R$ (is at time $R$ ) over the delay progress per cycle, i.e., $C-B$. If $C<B$, then there are three subcases. For the sake of simplicity, we assume that a Boolean expression that evaluates to true is equivalent to the numerical value one, and numerical value zero otherwise. In all three subcases, at least one cycle is required. If $t$ is less than or equal to $R-T$, then the beacon falls in the receive window in the first cycle. If $t$ is greater than $R-T$ and less than $C$, then the beacon is outside of the receive window. We must count the number of cycles required until $T$ falls within a receive window. This is determined by the ceiling of the ratio of the time difference between the left edge of $R$ and left edge of $T$ over the delay progress per cycle, i.e., $B-C$. Otherwise, $t$ is greater than or equal to $C$ and only one more cycle is required before the beacon falls within the receive window. The number of required cycles to get the beacon transmission $T$ in the receive window $R$ is $k_{t}=$

$$
\left\{\begin{array}{cl}
\left\lceil\frac{t+T-R}{C-B}\right\rceil+1 & : \quad C>B \\
1+(R-T<t<C) \cdot\left\lceil\frac{C-t}{B-C}\right\rceil+(C \leq t) & : \quad C<B
\end{array}\right.
$$

Assuming that $t$ is a random variable that follows an uniform distribution. Then $k_{t}$ is also a random variable, with uniform distribution, over the range $k_{0} \ldots k_{B}$. The expectation of $k_{t}$ is defined as:

$$
E\left[k_{t}\right]=\frac{1}{B} \sum_{t=0}^{B} k_{t}
$$

Hence, given $n$ channels to scan, the expectation of the persistent scanning time $S$ is:

$$
E[S]=n \cdot C \cdot E\left[k_{t}\right]
$$

Figure 5 plots the expectation of the sequential scanning time as a function of the duration of a cycle $C$. The x-axis ranges over the possible values of $C$, from $85 \mathrm{~ms}$ to $135 \mathrm{~ms}$ (chosen values are similar to the ones in Ref. [6]). The duration of the receive window $R$ is set to $30 \%$ of $C$, with a maximum of $40 \mathrm{~ms}$. The beacon period $B$ is $102.4 \mathrm{~ms}$. The beacon length $T$ is $0.5 \mathrm{~ms}$. It assumed that the total number of channels $n$ that needs to be scanned is 23 . The $y$-axis ranges over the possible values of the expected scanning time, in seconds. The expected value peaks when $C$ is close to $B$, i.e., $100 \mathrm{~ms}$ or $105 \mathrm{~ms}$. This is because when $T$ is off the receive window, it progresses slowly towards the receive window, from cycle to cycle, due to the small difference between $C$ and $B$. This is consistent with results described in Ref. [6].

\section{B. Sliding-Window Scanning}

As shown in Figure 6, when $C$ is equal to $B$ in duration sequential scanning may fail because the beacon never falls within the receive window. When $t$ is greater than or equal

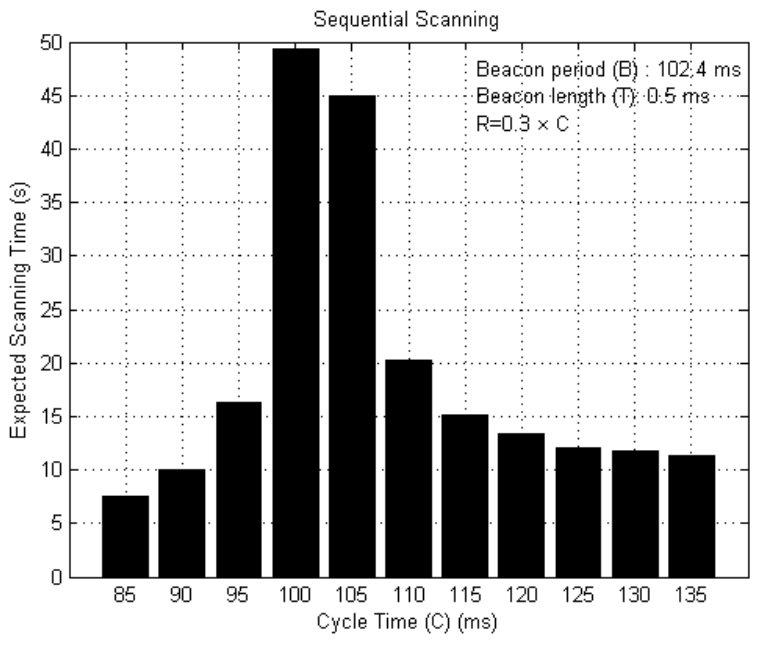

Fig. 5. Expected sequential scanning time: $C>B$ or $C<B$.

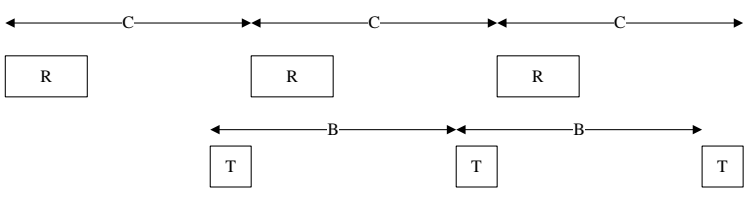

Fig. 6. Sequentoal scanning with $C=B$.

to zero and less than or equal to $R-T, T$ is within the receive window and only one cycle is required. Otherwise, $T$ falls outside and will always fall outside the receive window. Sequential scanning doesn't terminate.

The sliding-window strategy is designed to address the case $C$ equals $B$. The receive window relatively slides from the left edge of the receive cycle to the right edge, from cycle to cycle, until $T$ falls within $R$, see Figure 7. Each time it makes a relative displacement corresponding to $R$. As pictured in Figure 7, if $i \times R-T<t<i \times R(i=1,2,3, \ldots)$, then the beacons falls partly in a receive window. Sliding window fails. Receive windows must overlap by a time duration corresponding to $T$. If a beacon is partially covered by the end of a receive window, then it will fall entirely within the receive window of the next cycle.

We develop a probabilistic model of sliding-window scanning. The number of cycles until beacon transmission $T$ falls within the receive window $R$, as a function of $t$ is the ceiling of the ratio of $t$ over $R$ minus $T$, each cycle covers an additional $R-T$ of the period. The expectation of the number of cycles required is the summation of all values of $k_{t}$ over $B$. The number of cycles until beacon transmission $T$ falls within the receive window $R$, as a function of $t \in 0 \ldots B$ is:

$$
k_{t}=\left\lceil\frac{t}{R-T}\right\rceil
$$




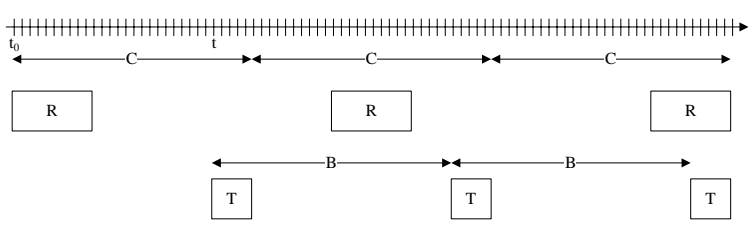

Fig. 7. Sliding-window scanning: $C=B$ (with no overlapping).

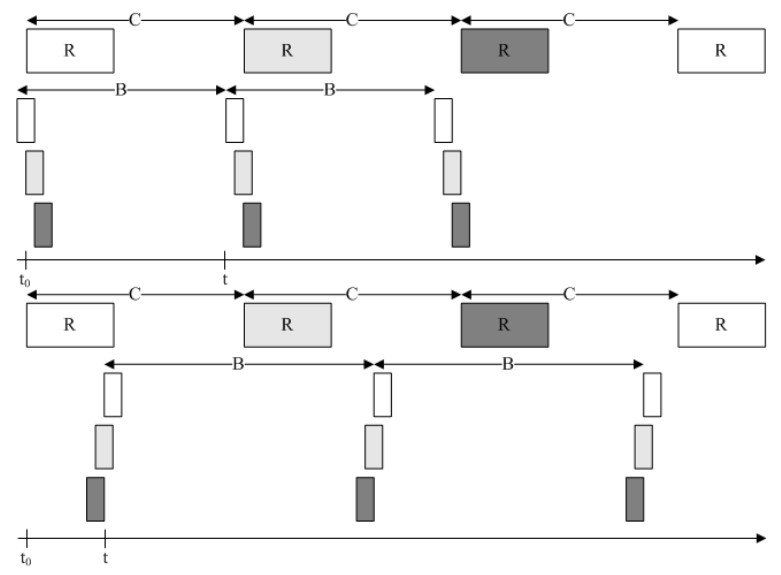

Fig. 8. Worst cases of pseudo-concurrent scanning: $C>B$ and $B>C$.

The expectation of $k_{t}$ is:

$$
E\left[k_{t}\right]=\frac{1}{B} \sum_{t=0}^{B} k_{t}
$$

\section{Pseudo-Concurrent Scanning}

Figure 8 pictures the worst case of pseudo-concurrent scanning, assuming $C>B$. The group size $m$ is three. The scanning rotation consists of the white, light gray and dark gray channels. During the first cycle, the white beacon partially overlaps with $R$. Success is not possible, even though the receiver is tuned to the white channel. Then similar conditions apply to the light gray and dark gray beacons, in the second and third cycles. The right edge of the dark gray beacon has to slide, relatively to $R$, from left to right until it falls within a receive window tuned to the dark gray channel. Then all beacons will be received.

We develop a probabilistic model of pseudo-concurrent scanning. For this case, an upper bound on the scanning time is obtained. That is, we analyze the worst cases. When $C>B$, the number of cycles is less than a ratio plus $m$. The ratio is the time delay between the right edge of the leading beacon, at time $t+T$ in the upper part of Figure 8, and the right edge of $R$ (is at time $R$ ) over the relative progress per cycle, i.e., $C-B$. Note that $t+T$ is inferior to $B+T$. Once the leading beacon falls within a receive windows, it remains in the window for at least $m$ cycles. Each beacon is received in one of the receive windows. If $C>B$, then we have that the number of cycles $k$ per group:

$$
k<\left\lceil\frac{B+T-R}{C-B}\right\rceil+m
$$

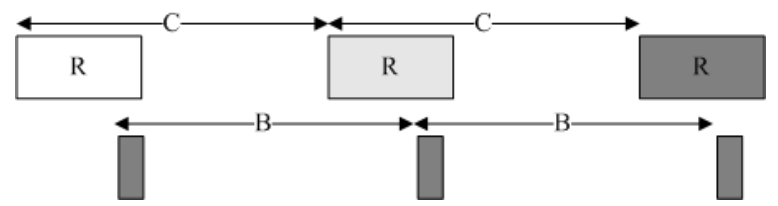

Fig. 9. Pseudo-concurrent scanning, selection of $m$ such that any beacon falls in at least one of $m$ consecutive receive windows $(C>B$ is assumed in this example).

The case $C<B$ is symmetric to the case $C>B$. The number of cycles is less than a ratio plus $m$. The ratio is the time delay between the left edge of the lagging beacon, at time $t$ in lower part of Figure 8, and the left edge of the first $R$ after $t$ (is at time $C$ ) over the relative progress per cycle, i.e., $B-C$. Note that $t$ is greater than $R-T$. Once the lagging beacon falls within a receive windows, it remains in the window for at least $m$ cycles. Each beacon is received in one of the receive windows. If $B>C$, then we have that the number of cycles $k$ per group:

$$
k<\left\lceil\frac{C+T-R}{B-C}\right\rceil+m
$$

The group size needs to be selected such that all beacons fall within at least $m$ consecutive receive windows, see Figure 9. Among these $m$ consecutive windows, the receiver is tuned to the channel of each beacon at least once and each beacon is heard. The selection of $m$ according to Equation 1 works, assuming $T \leq|C-B|$.

Worst case scanning time is the product of the number of groups, receive period and number of cycles:

$$
\left\lceil\frac{n}{m}\right\rceil \cdot C \cdot k
$$

Figure 10 plots the expectation of the worst case pseudoconcurrent time as a function of the duration of a cycle $C$. The $\mathrm{x}$-axis ranges over the possible values of $C$, from $85 \mathrm{~ms}$ to $135 \mathrm{~ms}$. The duration of the receive window $R$ is set to $30 \%$ of $C$, with a maximum of $40 \mathrm{~ms}$. For the sequential case, the diagram 5 plots the expected time while for the pseudoconcurrent case, the diagrams 10 plots an upper bound of the expected scanning time. Nevertheless, the plot reveals a substantial drop with pseudo-concurrent scanning time.

\section{Truly-Concurrent Scanning}

Truly-concurrent scanning can be combined with any of the aforementioned scanning schemes. We may assume that a device has enough non-interfering radio interfaces or bandwidth to scan simultaneously $l$ channels. Hence the expected scanning time is reduced by at least a factor corresponding to the ceiling of the ratio of $n$ over $l$. If sequential scanning is used, then $l$ channels can be simultaneously scanned. If pseudo-concurrent scanning is used, then $l$ groups can be simultaneously scanned. Pseudo-concurrency is applied within groups and true concurrency across groups. 


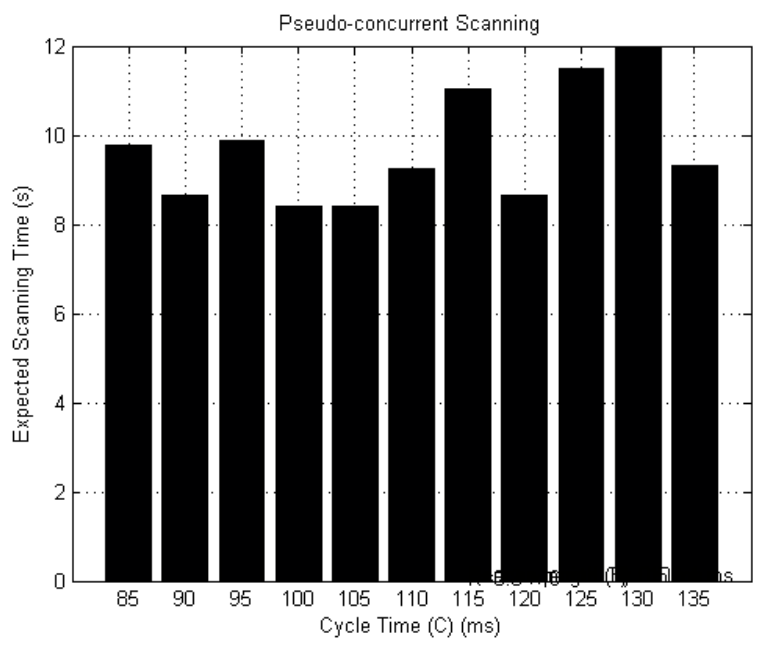

Fig. 10. Worst case pseudo-concurrent scanning: $C>B$ or $C<B$.

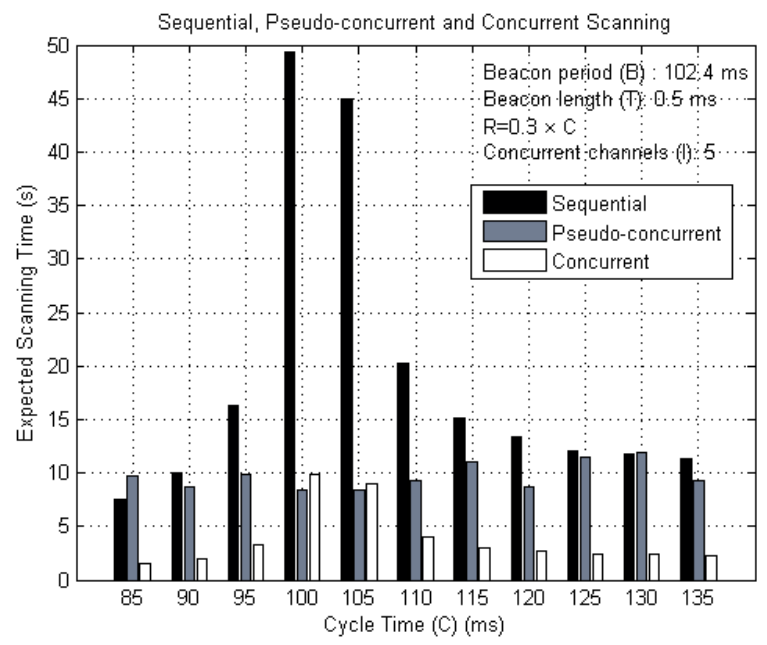

Fig. 11. Sequential, concurrent and pseudo-concurrent scanning comparison.

With $l$ concurrently scanned channels, scanning time $S$ is:

$$
E[S] \leq\left\lceil\frac{n}{l}\right\rceil \cdot C \cdot k
$$

where $k$ is the number of cycles required to scan one channel (with sequential scanning).

Figure 11 shows scanning time, in the case of sequential scanning (expected), pseudo-concurrent (worst case) and concurrent (expected). The number of channels $n$ is 23 . In the concurrent case, the number of concurrently scanned channels $l$ is 5 . In other words, five channels are concurrently sequentially scanned. Concurrent scanning is almost always better. When the difference between $C$ and $B$ is small, however, it is interesting to observe that pseudo-concurrent scanning can do better with less resources than five channel trulyconcurrent scanning. Sequential scanning, even in parallel, is slow because of small changes (determined by $|C-B|$ ) from one cycle to another.

\section{CONCLUSion}

We have analyzed four strategies to solve the scanning problem. We constructed probabilistic models for the sequential scanning and pseudo-concurrent scanning strategies, according to their definition in Ref. [6]. Consistently with results provided in Ref. [6], the expected value of sequential scanning time peaks when the receiver period is close to the broadcast period. This is because when the beacon is off, it progresses slowly towards the receive window, from cycle to cycle, due to the small difference between the receive period and broadcast period. When the receive period and broadcast period are equal, sequential scanning may fail because the beacon never falls within the receive window. We have designed the sliding-window strategy to address this case. With respect to sequential scanning, the analysis reveals a substantial drop with pseudo-concurrent scanning time. When hardware resources are available, truly-concurrent scanning can be combined with any of the sequential scanning, slidingwindow or pseudo-concurrent scanning strategies.

\section{REFERENCES}

[1] X. Jing and D. Raychaudhuri, "Spectrum co-existence of IEEE 802.11b and 802.16a networks using the CSCC etiquette protocol," in First IEEE International Symposium on New Frontiers in Dynamic Spectrum Access Networks (DySPAN), pp. 243-250, November 2005.

[2] J. Zhu and H. Yin, "Enabling collocated coexistence in IEEE 802.16 networks via perceived concurrency," IEEE Communications Magazine, vol. 47, pp. 108-114, June 2009.

[3] J. Zhu, A. Waltho, X. Yang, and X. Guo, "Multi-radio coexistence: Challenges and opportunities," in Proceedings of 16th International Conference on Computer Communications and Networks (ICCCN), pp. 358 364, August 2007.

[4] M. Konrad, W. Koch, and J. Huschke, "Coexistence analysis of Bluetooth and cellular UMTS in the 2500-2690 MHz band," in IEEE Wireless Communications and Networking Conference (WCNC), vol. 3, pp. 12451249, April 2006.

[5] C.-W. P. Huang, W. Vaillancourt, A. Parolin, C. Zelley, and G. Zeji, "A compact high rejection $2.4 \mathrm{GHz}$ WLAN front-end module enables multiradio co-existence up to $2.17 \mathrm{GHz}$," in IEEE Radio Frequency Integrated Circuits (RFIC) Symposium, p. 4, June 2006.

[6] J. Zhu and O. Markovits, "Techniques for detecting beacons on wireless channels - United States Patent Application 20100309893," December 2010 .

[7] "IEEE recommended practice for information technology - telecommunications and information exchange between systems - local and metropolitan area networks - specific requirements part 15.2: Coexistence of wireless personal area networks with other wireless devices operating in unlicensed frequency bands," IEEE Std 802.15.2-2003, 2003.

[8] A. Adya, P. Bahl, J. Padhye, A. Wolman, and L. Zhou, "A multi-radio unification protocol for IEEE 802.11 wireless networks," in Proceedings of the First International Conference on Broadband Networks, BROADNETS '04, (Washington, DC, USA), pp. 344-354, IEEE Computer Society, 2004.

[9] F. Xue and X. Yang, "Scheduling and coding for unicast in multi-radio systems: the two-user case," in Proceedings of the 4th Annual International Conference on Wireless Internet, WICON '08, (ICST, Brussels, Belgium, Belgium), pp. 46:1-46:8, ICST (Institute for Computer Sciences, Social-Informatics and Telecommunications Engineering), 2008. 